\title{
Partial Volume Effect on MR Elastography
}

\author{
Daiki Ito1,2,3, Tomokazu Numano, ${ }^{1 *}$, Kazuyuki Mizuhara ${ }^{2,4}$, Toshikatsu Washio², \\ Masaki Misawa' ${ }^{2}$, Naotaka Nitta ${ }^{2}$ \\ ${ }^{1}$ Department of Radiological Science, Graduate School of Human Health Science, Tokyo Metropolitan University, Tokyo, Japan \\ ${ }^{2}$ Health Research Institute, National Institute of Advanced Industrial Science and Technology, Tsukuba, Japan \\ ${ }^{3}$ Office of Radiation Technology, Keio University Hospital, Tokyo, Japan \\ ${ }^{4}$ Department of Mechanical Engineering, Tokyo Denki University, Tokyo, Japan \\ Email: ${ }^{\star}$-numano@tmu.ac.jp
}

How to cite this paper: Ito, D., Numano, T., Mizuhara, K., Washio, T., Misawa, M. and Nitta, N. (2017) Partial Volume Effect on MR Elastography. Open Journal of Medical Imaging, 7, 131-143. https://doi.org/10.4236/ojmi.2017.74013

Received: September 8, 2017

Accepted: September 27, 2017

Published: September 30, 2017

Copyright (c) 2017 by authors and Scientific Research Publishing Inc. This work is licensed under the Creative Commons Attribution International License (CC BY 4.0).

http://creativecommons.org/licenses/by/4.0/

\begin{abstract}
Magnetic resonance elastography (MRE) allows the quantitative assessment of the stiffness of tissues based on the tissue response to oscillatory shear stress. Shear wave displacements of the tissues are encoded as phase shifts and converted to stiffness (elastogram). Generally, a partial volume effect occurs when different materials are encompassed on the same voxel. In MRE, however, the partial volume effect occurs even if the voxel is filled with the same materials because wave displacements due to vibrations are spatially distributed. The purpose of this study was to investigate how the partial volume effect can affect the phase shift and the elastogram in MRE. We assumed that the partial volume effect appears only in the slice thickness direction and performed a simulation and MRE experiment with various slice thicknesses (1 - $19 \mathrm{~mm})$, two types of imaging plane (coronal and axial) and two types of vibration frequency $(100$ and $200 \mathrm{~Hz}$ ). The results of the simulation and the MRE experiment were similar, and indicated that the phase shift and the elastogram changed variously depending on the slice thickness, the wave pattern and the vibration frequency, even if the voxel was filled with the same material. To reduce the partial volume effect, it is necessary to perform the MRE under the following conditions: Use a wave pattern which barely causes this artefact, a smaller voxel size and a lower vibration frequency.
\end{abstract}

\section{Keywords}

Magnetic Resonance Elastography, Partial Volume Effect, Artefact, Elasticity, Viscoelasticity

\section{Introduction}

Manual palpation has long been used clinically to diagnose abnormal stiffness 
changes in soft tissue. However, the technique is subjective and deep or small tissues are often inaccessible. Accordingly, elastography techniques have been developed to image the mechanical properties of tissues by using ultrasound [1] [2] and magnetic resonance imaging (MRI) [3] [4]. Dynamic MR elastography (MRE) is a phase-contrast MR technique that is capable of assessing the mechanical properties of tissues through the visualization and analysis of harmonic shear vibrations. Then, MRE can be performed on both superficial and deep tissues only if vibrations reach these tissues. In MRE, tissue displacements due to vibrations cause a phase shift of nuclear spin and are encoded in a phase image (wave image) by using bipolar gradient lobes (motion encoding gradient: MEG). Moreover, recorded wave images are enhanced by processing using a variety of methods (e.g., phase unwrapping, band pass filtering and noise suppression) [5]. Estimates of the local shear wavelengths in the tissue using the wave image provide stiffness images (elastograms). In vivo MRE has been successfully applied to reveal the stiffness of a variety of human tissues and organs such as the brain [4] [6], breast [4] [7], liver [4] [8], prostate [9] [10], and skeletal muscles [4] [10].

A partial volume effect is an imaging artefact associated with many imaging modalities. It is well known that the partial volume effect yields average arbitrary values (e.g., the Hounsfield unit in computed tomography) of the materials within a voxel. Therefore, the partial volume effect does not occur when the voxel is filled with the same materials in many imaging techniques. In MRE, however, the partial volume effect occurs in wave images even if the voxel is filled with the same materials because wave displacements due to vibrations are spatially distributed. The purpose of this study was to investigate how the partial volume effect can affect MRE images (wave images and elastograms). However, it is difficult to generalize the partial volume effect on MRE because the patterns of wave propagation depend on the excitation method, imaging plane and imaging object. Therefore, in this study, the MRE experiment was conducted first, then a simulation was conducted based on obtained wave patterns in the MRE experiment.

\section{Theory}

\subsection{Dynamic Phase-Contrast MRE}

Measuring tissue motion due to vibrations with MRE is based on an MR imaging technique called phase-contrast MRI [12]. Muthupillai et al. [3] developed the technique of dynamic phase-contrast MRE where propagating harmonic shear waves in tissue are encoded into a phase image by using bipolar gradient lobes (motion encoding gradient: MEG). When the period of the vibrations $T$ matched the duration of MEG $\delta$, the phase shifts due to vibrations is maximum.

First, consider the specific case in which the nuclear spins undergo harmonic motion about their mean position $r$

$$
\varepsilon(\boldsymbol{r}, \theta)=\boldsymbol{\varepsilon}_{0} \sin (\boldsymbol{k} \cdot \boldsymbol{r}-\omega t+\theta)
$$


where $k$ is the wave number vector, $\omega$ is the angular frequency of vibrations, $t$ is the time from the initiation of vibrations, $\theta$ is an initial phase offset, and $\varepsilon_{0}$ is the peak amplitude of motion vector. If a gradient waveform is rectangular with negligible ramp time, with the duration $(\delta=T)$, and harmonic motion is induced in tissue (Equation (1)), the observed phase shift [3] in the MR signal is given by

$$
\varphi(\boldsymbol{r}, \theta)=\frac{2 \gamma N T\left(\boldsymbol{G} \cdot \boldsymbol{\varepsilon}_{0}\right)}{\pi} \cos (\boldsymbol{k} \cdot \boldsymbol{r}+\theta)
$$

where $\gamma$ is the proton gyromagnetic ratio, $N$ is the number of MEG pairs used to sensitize the motion, and $G$ is the MEG vector. The initial phase offset $\theta$ also corresponds to the phase offset between the vibration and the MEG. This equation indicates that the phase shifts of harmonically vibrating nuclear spin are directly proportional to their displacement regardless of $\omega$ and $t$.

\subsection{Partial Volume Effect on MRE}

We assumed that a uniform object was subjected to a harmonic planar shear wave, which is attenuated as it propagates. In general, in-plane resolution is higher than that of the slice thickness direction. Hence, the partial volume effect of in-plane was ignored. If all nuclear spins of the imaging object have the same dimension magnetization vector, the phase shift due to a planar shear wave considering the slice thickness in a wave image is given by

$$
\Phi(\boldsymbol{r}, \theta)=\arctan \left(\frac{\int_{\boldsymbol{r}-\Delta s_{s t}}^{r+\Delta r_{s t}} \operatorname{Im}\left\{\varphi(\boldsymbol{r}, \theta) \exp \left(-\alpha_{s t} \lambda_{s t}\right)\right\} \mathrm{d} \boldsymbol{r}}{\int_{\boldsymbol{r}-\Delta \Delta_{s t}}^{r+\Delta r_{s t}} \operatorname{Re}\left\{\varphi(\boldsymbol{r}, \theta) \exp \left(-\alpha_{s t} \lambda_{s t}\right)\right\} \mathrm{d} \boldsymbol{r}}\right)
$$

where $\lambda_{s t}$ is the wavelength in the slice thickness direction, $\alpha_{s t}$ is the attenuation coefficient per wavelength in the slice thickness direction, $2\left|\Delta r_{s t}\right|$ is the slice thickness.

\section{Materials and Methods}

\subsection{Experimental Setup}

All MRE experiments were performed using a 2.0-T animal experiment MR scanner system (Biospec 20/30, BRUKER, Karlsruhe, Germany) with a B-GA20 Gradient System (maximum gradient strength of $\pm 100 \mathrm{mT} / \mathrm{m}$ ) and a 140 - $\mathrm{mm}$-ID birdcage coil tuned to $85 \mathrm{MHz}$ for proton resonance. The MR data acquisition, image reconstruction and pulse sequence development were performed with the ParaVision 4.0 (BRUKER) software system. A self-built waveform generation system (LabVIEW, USB-6221; National Instruments, TX, USA) was used to generate the vibration waveforms. This system was triggered to produce synchronized sinusoidal waves, which were power amplified and input a pneumatic pressure generator. Vibration of frequency 100 or $200 \mathrm{~Hz}$ generated this system were supplied to a vibration pad through a hose. Our MRE experiment was used the columnar shape phantom, which was made of acrylamide and has a diameter 
of $48.4 \mathrm{~mm}$ and a height of $90.8 \mathrm{~mm}$ [13]. The vibration pad was designed suitable forms for the phantom by using a three-dimensional (3D) printer (3D touch; 3D System, SC, USA) (Figure 1). In this MRE system, the vibration phase offset was controlled by the waveform generator, giving continuous (steady state) vibrations throughout the whole acquisition (each imaging period).

\subsection{Pulse Sequence and Data Acquisition}

MRE acquisitions in this study were performed with a self-modified spin echo (SE) MRE sequence. This sequence combines motion encoding gradients (MEG) with a spin echo imaging module. In this sequence, the MEG was applied symmetrically around a refocusing (180 degree RF) pulse and the period of external vibration and the period of MEG were in agreement.

In this study, we selected two imaging planes. One was parallel to the propagating waves (coronal image) and the other was perpendicular to it (axial image). The coronal image was taken at the center of the phantom and the axial image was taken near the vibration pad because the partial volume effect was evaluated in the region where the wave propagates to the phantom sufficiently (Figure 2(a)). The parameters in both images were set almost the same (Table 1) and the slice thickness was changed in the range of 1 to $19 \mathrm{~mm}$, with a $2 \mathrm{~mm}$ step. The MEG direction was set left-right (LR) to visualize a planar shear wave pattern to the wave images [14]. Our MRE experiment was also performed under the assumption that the partial volume effect appears only in the slice selected direction (slice thickness direction) because of high in-plane resolution.

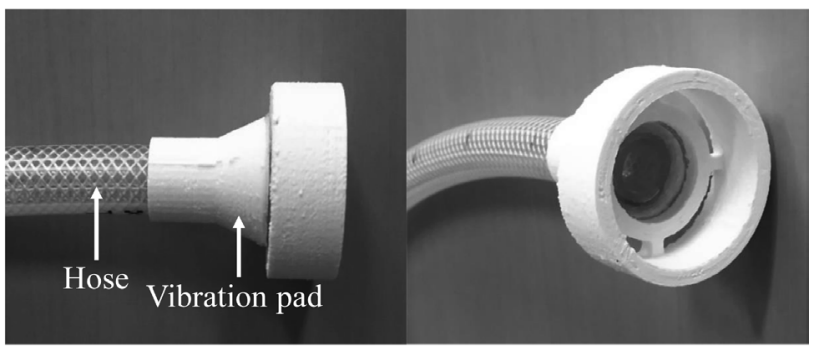

(a)

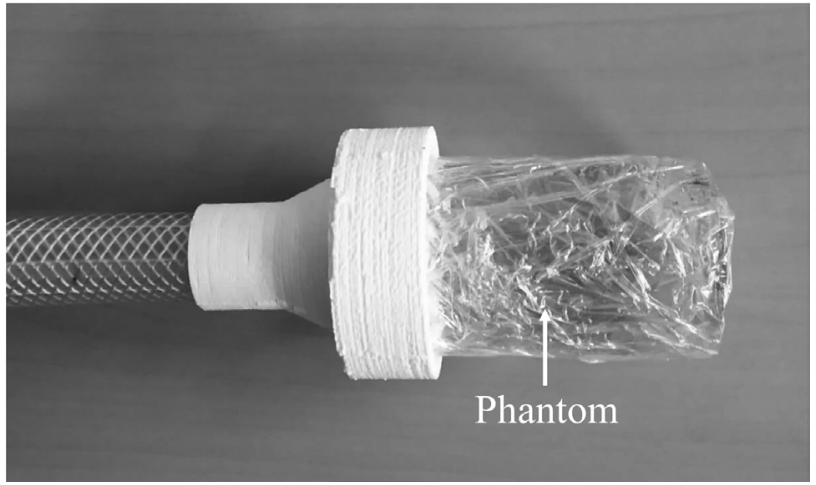

(b)

Figure 1. (a) Vibration pad; (b) Setup of the MRE experiment. 




Figure 2. (a) Schematic view of the MRE experiment and imaging planes (coronal and axial images); (b) Examples of coronal and axial wave images in the MRE experiment at $200 \mathrm{~Hz}$. Two white dotted lines indicate the profiles (R-Profile and L-Profile). Yellow frame and circle indicate the regions of interest (ROI); (c) Examples of coronal and axial wave images in the simulation.

Table 1. The MRE experiment parameters.

\begin{tabular}{ccc}
\hline Parameter & Coronal image & Axial image \\
Vibration freq. (Hz) & 100 or 200 & 100 or 200 \\
Vibration phase offset (radian) & $4(\pi / 2)$ & $4(\pi / 2)$ \\
Number of MEG pairs & One or two & One or two \\
MEG amplitude (mT/m) & 20 or 35 & 20 or 35 \\
MEG direction & Left-Right (LR) & Left-Right (LR) \\
Matrix & $128 \times 64$ & $64 \times 64$ \\
FOV (mm $\left.{ }^{2}\right)$ & $128 \times 64$ & $64 \times 64$ \\
Number of excitations & 1 & 1 \\
FA (degree) & 90 & 40 \\
TR (ms) & 400 & 40 \\
TE (ms) & 40 & $1-19$ \\
Slice thickness (mm) & $1-19$ & 25.6 \\
Total acquisition time (s) & 25.6 & 400
\end{tabular}

MEG: Motion Encoding Gradient, FOV: Field Of View, FA: Flip Angle.

\subsection{Image Processing and Data Analysis}

All wave images were processed by a phase unwrapping and a Gaussian spatial filter, and those were converted into elastograms which were processed by Local Frequency Estimate (LFE) algorithm freeware (MRE/Wave, MAYO CLINIC). 
For evaluating the change in the phase shift (amplitude of the shear wave) and stiffness image (elastogram) due to the slice thickness, the regions of interest (ROI) were set in the region of clear wave propagation (Figure 2(b) and Figure $2(d)$ ). The region of clear wave propagation was also different at each frequency $(100$ and $200 \mathrm{~Hz})$ because the wavelength was different at each frequency. Therefore, in this study, four ROIs were used according to each imaging plane and frequency. The change in the phase shift due to the slice thickness in the ROIs was evaluated by using the wave amplitude images which were obtained by a discrete Fourier transformation on the time series data (4 phase offset images). On the other hand, the change in the elastogram due to the slice thickness in the ROIs was evaluated by using Pearson's correlation. In this study, it was assumed that there is no partial volume effect at a $1 \mathrm{~mm}$ slice thickness, and we compared with elastograms at a $1 \mathrm{~mm}$ slice thickness and those of 3 - $19 \mathrm{~mm}$ slice thickness. The Pearson's correlation coefficient was determined using voxel-wise data for elastograms in the ROIs for each imaging plane and frequency. Significant difference was not evaluated because the sample number (pixel number in the ROI) was enormous.

\subsection{Simulation Parameters}

Our simulation was performed for wave patterns obtained in the MRE experiment by using Equation (3). To perform this simulation, it was necessary to measure the phase shift, the wavelength and the attenuation coefficient per wavelength in the slice thickness direction, in addition to the slice thickness. Moreover, in our simulation, the change in the phase shift in the direction perpendicular to wave propagation was ignored. This direction corresponded to anterior-posterior (AP) and left-right (LR) directions. In this study, as the slice thickness direction in the coronal image was the AP direction, the wave pattern, the phase shift and the elastogram in the coronal image was independent of the slice thickness in our simulation. In axial images, we assumed that the partial volume effect does not occur for a $1 \mathrm{~mm}$ slice thickness, and the phase shift, the wavelength and the attenuation coefficient per wavelength in the slice thickness direction were measured in that case. The phase shifts in axial images were recorded by the axial image at a $1 \mathrm{~mm}$ slice thickness by using the same ROIs as the MRE experiment. On the other hand, the wavelength and the attenuation coefficient per wavelength in the slice thickness direction require measuring in an orthogonal imaging plane. Thus, those values in the axial image were recorded by the coronal images at a $1 \mathrm{~mm}$ slice thickness by using two profiles (R-Profile and L-Profile), which were chosen manually along the shear wave propagation (Figure 2(b)). The wavelengths were calculated from the distance between peaks, and the averaged value in both profiles was recorded. The attenuation coefficients were also calculated from approximating peak amplitudes to exponential function. Then, the phase shift and the Pearson's correlation coefficient of the elastogram at each slice thickness were calculated by the same method used in the MRE experiment. 


\section{Results}

In coronal images, when the slice thickness was $1 \mathrm{~mm}$, measured wavelengths were about $12.0 \mathrm{~mm}$ and $5.63 \mathrm{~mm}$ for 100 and $200 \mathrm{~Hz}$, respectively. Measured attenuation coefficients per wavelength were also about 0.013 and 0.036 for 100 and $200 \mathrm{~Hz}$, respectively. In axial images, when the slice thickness was $1 \mathrm{~mm}$, measured mean phase shifts were about 1.280 and $0.531 \mathrm{rad}$ for 100 and $200 \mathrm{~Hz}$, respectively.

Figure 3 shows the coronal and axial wave images in 100 and $200 \mathrm{~Hz}$, for 1, 7, 13 and $19 \mathrm{~mm}$ slice thicknesses. In the case of the $1 \mathrm{~mm}$ slice thickness, the obtained wave pattern was a conventional pattern with pneumatic drivers, where the pattern is two out-of-phase wave lobes antisymmetric about the central axis in the LR direction [14]. Our simulation was performed for this wave pattern (Figure 2(c) and Figure 2(e)). Chroma of the wave images also indicates the phase shifts (wave displacements) due to the propagating shear wave in the phantom, and the phase shifts were normalized with that of a $1 \mathrm{~mm}$ slice thickness at each imaging plane and vibration frequency. At first glance, the phase shifts were barely changed by the slice thickness in coronal images, however, these decreased as the slice thickness increased in axial image. Moreover, the pattern of the wave images was changed as the slice thickness increased in axial images. In the simulation, the pattern of the wave images was unchanged due to the slice thickness, although the wave images were not shown in the figure.

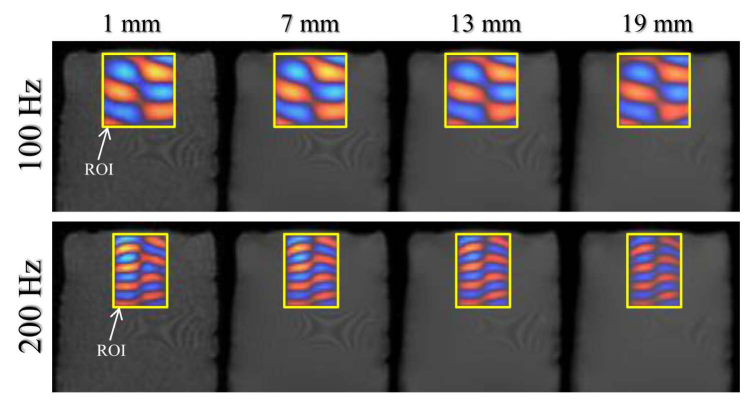

Coronal wave image fusion

(a)

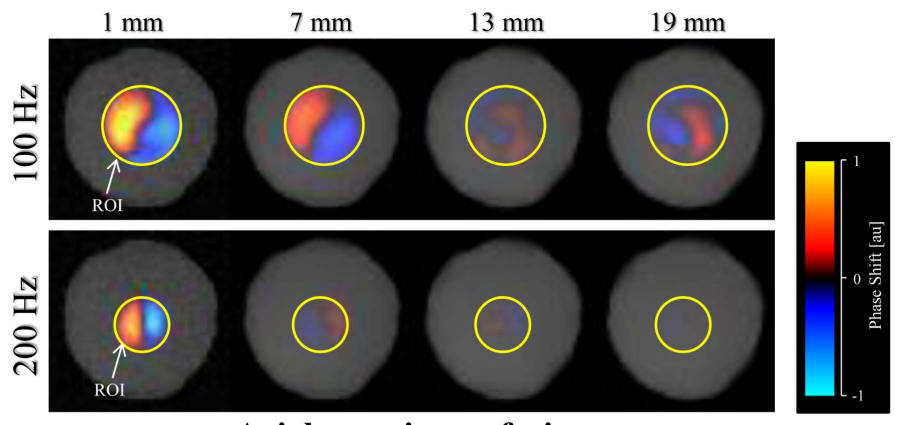

Axial wave image fusion

(b)

Figure 3. Coronal (a) and axial (b) magnitude images overlaid with the wave images for 100 and $200 \mathrm{~Hz}$ vibration at each slice thickness (1, 7, 13 and $19 \mathrm{~mm}$ ). 
Figure 4(a) shows the relative mean phase shifts of the coronal images for 100 and $200 \mathrm{~Hz}$ in the MRE experiment/simulation, normalized with that of $1 \mathrm{~mm}$ slice thickness, as a function of the slice thickness. In the simulation, the phase shifts were independent of the slice thickness in both frequencies. However, in the MRE experiment, the phase shifts decreased gradually with increasing slice thickness, and that of the change at $200 \mathrm{~Hz}$ was slightly larger than at $100 \mathrm{~Hz}$. Figure 4(b) shows the relative mean phase shifts of the axial image, for 100 and $200 \mathrm{~Hz}$ in the MRE experiment/simulation, normalized with that of $1 \mathrm{~mm}$ slice thickness, as a function of the slice thickness. In the simulation, the phase shifts decreased rapidly with increasing slice thickness until about one-wavelength (12.0 and $5.63 \mathrm{~mm}$ in 100 and $200 \mathrm{~Hz}$, respectively) and then repeated cyclic ups and downs. The phase shifts at $200 \mathrm{~Hz}$ also decreased rapidly compared with that at $100 \mathrm{~Hz}$ because the wavelength at $200 \mathrm{~Hz}$ was shorter than that of $100 \mathrm{~Hz}$. In the MRE experiment, the change trend in the phase shifts was very similar to that of the simulation. The phase shifts over one-wavelength decreased about $90 \%$ as compared with that of $1 \mathrm{~mm}$.

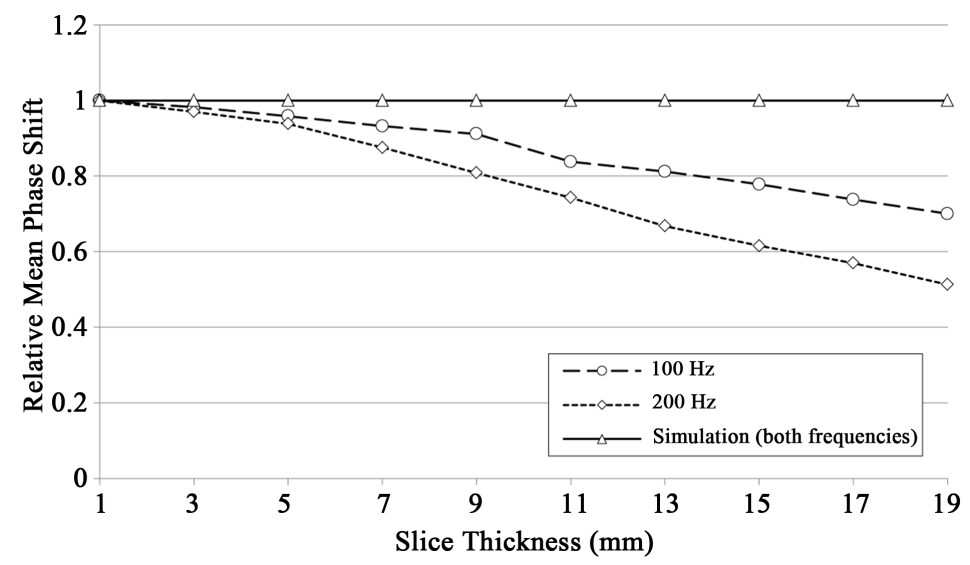

(a)

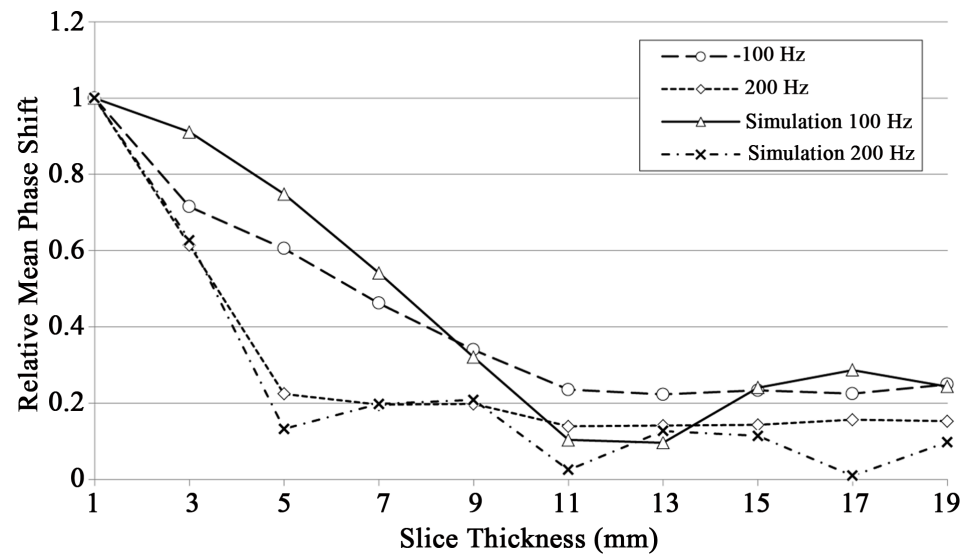

(b)

Figure 4. Relative mean phase shift (normalized with that of a $1 \mathrm{~mm}$ slice thickness) in the MRE experiment/simulation for 100 and $200 \mathrm{~Hz}$ vs. slice thicknesses, in the coronal (a) and axial (b) images. 
Figure 5(a) and Figure 5(b) show the Pearson's correlation coefficients in the coronal and axial images, respectively, as a function of the slice thickness. The Pearson's correlation coefficients were calculated between $1 \mathrm{~mm}$ slice thickness and $3-19 \mathrm{~mm}$ slice thickness for 100 and $200 \mathrm{~Hz}$ vibrations in the MRE experiment/simulation. In the simulation, the Pearson's correlation coefficients of both imaging planes and frequencies were independent of the slice thickness, in other words, the stiffness was independent of the slice thickness. However, in the MRE experiment, the Pearson's correlation coefficients decreased with increasing the slice thickness and those of the change trend were different in imaging planes and frequencies. In the coronal images (Figure 5(a)), the Pearson's correlation coefficients in both frequencies decreased slightly with increasing slice thickness, and that of the change at $200 \mathrm{~Hz}$ was larger than at $100 \mathrm{~Hz}$, such as in Figure 4(a). In the axial images (Figure 5(b)), the Pearson's correlation coefficients in both frequencies were high values (more than 0.7 ) and were not changed much by the slice thickness until about one-wavelength; however, over about one-wavelength, the Pearson's correlation coefficients in both frequencies decreased rapidly, and then repeated ups and downs.

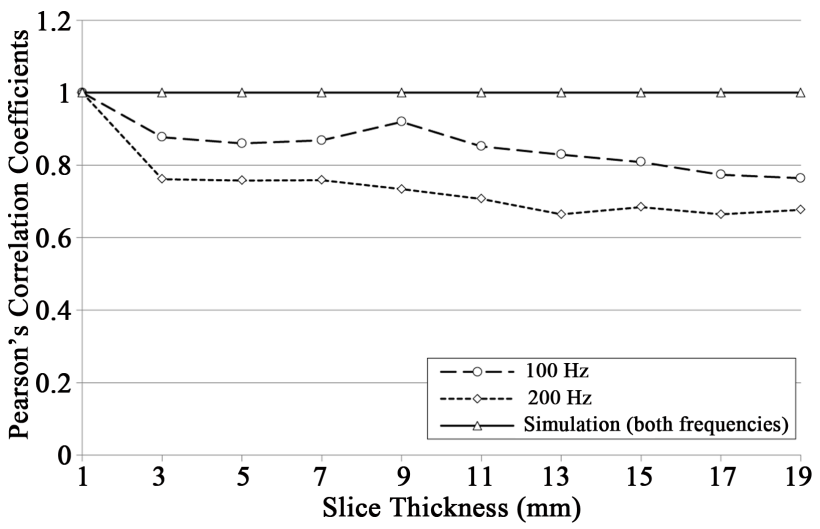

(a)

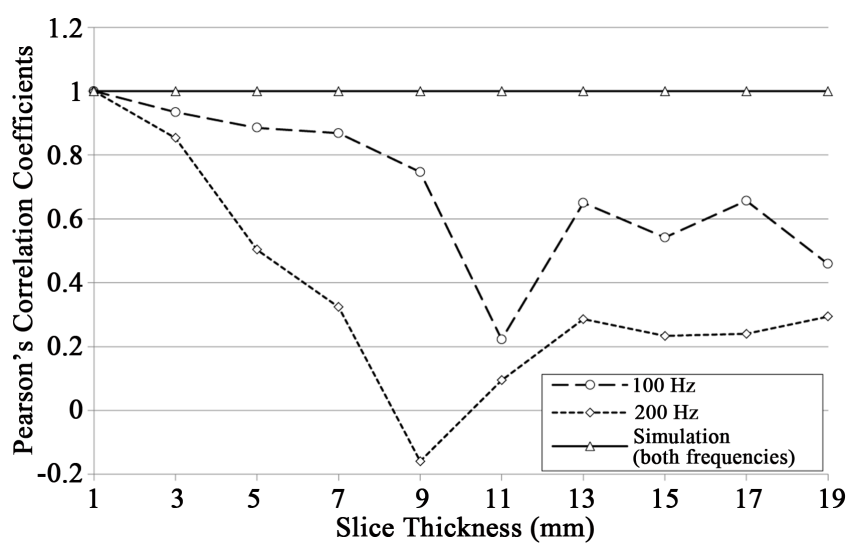

(b)

Figure 5. Pearson's correlation coefficients (normalized with that of a $1 \mathrm{~mm}$ slice thickness) in the MRE experiment/simulation for 100 and $200 \mathrm{~Hz}$ vs. slice thicknesses, in the coronal (a) and axial (b) images. 


\section{Discussion}

The results demonstrate that the phase shift (wave amplitude) and the stiffness change under the influence of the partial volume effect. In this study, overall change trend of the phase shift and the Pearson's correlation coefficients of the elastograms due to the slice thickness were similar in the simulation and the MRE experiment. However, there were mismatches between them.

As for the mismatch in the phase shift changes due to the slice thickness, for coronal images, the phase shifts decreased slightly with increasing slice thickness while those of the simulation were unchanged, and this mismatch was large at higher frequencies (Figure 4(a)). The reason for this discrepancy may be the change in the phase shift in the slice thickness direction (AP direction) in coronal images. In our simulation, we assumed that there is no change in the phase shift in the AP and LR directions because this simulation was based on the premise that planar shear waves were propagating to the imaging object. In our MRE experiment, obtained wave patterns were two out-of-phase wave lobes antisymmetric about the central axis and these wave lobes were close to a planar wave pattern. However, the phase shifts slightly decreased in AP direction as the distance becomes larger from the vibration source (Figure 3(b)). This change in the phase shift in AP direction may cause the partial volume effect, which leads to a reduction in the phase shift with increasing slice thickness in coronal images. Moreover, at $200 \mathrm{~Hz}$, as the wavelength was shorter and vibration penetration ability was lower than those at $100 \mathrm{~Hz}$, decreasing phase shifts due to the distance from the vibration source were larger than $100 \mathrm{~Hz}$. Therefore, the partial volume effect at $200 \mathrm{~Hz}$ may be large compared with that of $100 \mathrm{~Hz}$.

MRE is a special technique which assesses the mechanical properties of tissues quantitatively. However, errors when using MRE may cause a misdiagnosis. In this study, the pattern of the wave images and the Pearson's correlation coefficients of the elastograms were changed due to the slice thickness, although those of the simulation were unchanged. We speculate that these changes were because of reflection waves from the boundaries. The wave interference because of the reflection waves may lead to wave image changes, and this change may affect the elastogram. As compared with the Pearson's correlation coefficients (Figure 5) and the phase shifts (Figure 4), the Pearson's correlation coefficients were decreased when the phase shifts became small. It is thus assumed that small phase shifts may relatively enhance the influence of the reflected waves. Especially with regards to in vivo MRE, it is very difficult to propagate even planar shear waves into the tissues because the reflection wave is likely to be generated from the boundaries of a variety of tissues. Hence, the influence of the partial volume effect in vivo MRE may be larger than that in our MRE experiment. Therefore, we assumed that there are 3 methods to reduce this artefact based on the results.

First, performing the MRE in the imaging plane and motion encoding direction are less likely to cause the partial volume effect. These decide the wave pattern in the wave image. In this study, the axial image was a wave pattern where 
the partial volume effect easily occurs because the slice selective direction was parallel to the wave propagated direction. On the other hand, the coronal image was a wave pattern where the partial volume effect hardly occurred because the slice selective direction was perpendicular to the wave propagated direction. However, even in the coronal image of our MRE experiment, the MRE images were affected by the partial volume effect because of attenuating wave amplitudes and the reflection waves. As mentioned the above, there is a possibility that the influence of the partial volume effect because of reflection waves may be larger in the in vivo MRE. Hence, the wave pattern (imaging plane and motion encoding direction) should be carefully selected to avoid the partial volume effect.

Second, we looked at performing the MRE with thinner slice thicknesses (smaller voxels). In both the simulation and MRE experiment, the phase shift decreased with increasing slice thickness. Therefore, the MRE was performed for thinner slice thicknesses (smaller voxel sizes) to obtain a large phase shift due to the wave displacement. In the MRE, a phase-to-noise ratio (PNR) in the MR phase signal affects the image quality, where the PNR is proportional to the SNR and to the phase shift [15]. Therefore, the combination of a large phase shift and a high SNR (thick slice thickness) are required to obtain an image with higher PNR. However, these may be difficult because a thick slice thickness leads to a small phase shift due to the partial volume effect. Considering the influence of the reflection waves, it may be better to set the slice thickness as thin as possible.

Third, we considered performing the MRE at lower vibration frequencies. The partial volume effect is stronger at higher vibration frequencies because it depends on the wavelength of the propagating wave in an imaging object. The wavelength also depends not only on vibration frequency, but also the stiffness of the imaging object since the wavelength becomes shorter in softer objects. Thus, the partial volume effect would be stronger in the case of a high vibration frequency and a soft object. Currently, the vibration frequency is chosen as a tradeoff between penetration depth and stiffness resolution. In the future, the vibration frequency may be chosen considering not only the penetration depth and stiffness resolution, but also the partial volume effect. Moreover, the MRE is used for measuring not only the elasticity, but also the viscoelasticity, in tissues in vivo [16] [17]. To measure the viscoelasticity, it is necessary to measure multiple wave amplitudes at multiple vibration frequencies. The partial volume effect causes a change in the amplitude depending on the wavelength of the propagating wave. Therefore, in the case of viscoelasticity measurement, the partial volume effect may exert larger influences and easily result in misdiagnosis.

One limitation of this study is that the partial volume effect was investigated by using simple wave patterns. In this study, the influence of the partial volume effect was investigated in planar shear waves propagating. However, the influence of the partial volume effect may change in different wave patterns, such as spherical shear waves. Second, the influence of the partial volume effect in vivo was not investigated. In the in vivo MRE, we suspect that the wave image would 
become a complex wave pattern as compared with that of our MRE experiment. A complex wave may cause unexpected partial volume effect.

\section{Conclusion}

This study demonstrated both theoretically and experimentally that the phase shift (wave amplitude) was changed by the partial volume effect. Moreover, in the MRE experiment, the stiffness changed by the partial volume effect because of reflection wave. The partial volume effect may cause a misdiagnosis because this artefact does not cause a noticeable change in the image, such as an aliasing artefact. Moreover, this artefact depends on the following parameters: wave pattern (imaging plane and motion encoding direction), voxel size, and vibration frequency. To reduce this artefact, we suggested that the wave pattern should be considered first, then reducing the voxel size and the vibration frequency because reduced voxel size leads to lower PNR of the MR phase signal and reduced vibration frequency lowers the resolution of the elastogram. The MRE is a novel noninvasive technique, which is capable of providing quantitative information on the mechanical properties of tissues, which can provide significant diagnostic potential. Conversely, MRE errors may cause a misdiagnosis. To reduce MRE errors, further basic investigation is needed.

\section{References}

[1] Sandrin, L., Tanter, M., Gennisson, J.L., Catheline, S. and Fink, M. (2002) Shear Elasticity Probe for Soft Tissues with 1-D Transient Elastography. IEEE Transactions on Ultrasonics, Ferroelectrics, and Frequency Control, 49, 436-446. https://doi.org/10.1109/58.996561

[2] Sarvazyan, A.P., Rudenko, O.V., Swanson, S.D., Fowlkes, J.B. and Emelianov, S.Y. (1998) Shear wave elasticity imaging: a new ultrasonic technology of medical diagnostics. Ultrasound in Medicine \& Biology, 24, 1419-1435. https://doi.org/10.1016/S0301-5629(98)00110-0

[3] Muthupillai, R., Lomas, D.J., Rossman, P.J., Greenleaf, J.F., Manduca, A. and Ehman, R.L. (1995) Magnetic Resonance Elastography by Direct Visualization of Propagating Acoustic Strain Waves. Science, 269, 1854-1857. https://doi.org/10.1126/science.7569924

[4] Mariappan, Y.K., Glaser, K.J. and Ehman, R.L. (2010) Magnetic Resonance Elastography: A Review. Clinical Anatomy, 23, 497-511. https://doi.org/10.1002/ca.21006

[5] Li, B.N., Shan, X., Xiang, K., An, N., Xu, J., Huang, W., et al. (2014) Evaluation of Robust Wave Image Processing Methods for Magnetic Resonance Elastography. Computers in Biology and Medicine, 54, 100-108. https://doi.org/10.1016/j.compbiomed.2014.08.024

[6] Fattahi, N., Arani, A., Perry, A., Meyer, F., Manduca, A., Glaser, K., et al. (2016) MR Elastography Demonstrates Increased Brain Stiffness in Normal Pressure Hydrocephalus. American Journal of Neuroradiology, 37, 462-467. https://doi.org/10.3174/ajnr.A4560

[7] Siegmann, K.C., Xydeas, T., Sinkus, R., Kraemer, B., Vogel, U. and Claussen, C.D. (2010) Diagnostic Value of MR Elastography in Addition to Contrast-Enhanced MR Imaging of the Breast-Initial Clinical Results. European Radiology, 20, 318-325. https://doi.org/10.1007/s00330-009-1566-4 
[8] Yoshimitsu, K., Mitsufuji, T., Shinagawa, Y., Fujimitsu, R., Morita, A., Urakawa, H., et al. (2016) MR Elastography of the Liver at 3.0 T in Diagnosing Liver Fibrosis Grades; Preliminary Clinical Experience. European Radiology, 26, 656-663. https://doi.org/10.1007/s00330-015-3863-4

[9] Li, S., Chen, M., Wang, W., Zhao, W., Wang, J., Zhao, X., et al. (2011) A Feasibility Study of MR Elastography in the Diagnosis of Prostate Cancer at 3.0T. Acta Radiologica, 52, 354-358. https://doi.org/10.1258/ar.2010.100276

[10] Sahebjavaher, R.S., Nir, G., Honarvar, M., Gagnon, L.O., Ischia, J., Jones, E.C., et al. (2015) MR Elastography of Prostate Cancer: Quantitative Comparison with Histopathology and Repeatability of Methods. NMR in Biomedicine, 28, 124-139. https://doi.org/10.1002/nbm.3218

[11] Ito, D., Numano, T., Mizuhara, K., Takamoto, K., Onishi, T. and Nishijo, H. (2016) A New Technique for MR Elastography of the Supraspinatus Muscle: A Gradient-Echo Type Multi-Echo Sequence. Magnetic Resonance Imaging, 34, 1181-1188.

[12] Moran, P.R. (1982) A Flow Velocity Zeugmatographic Interlace for NMR Imaging in Humans. Magnetic Resonance Imaging, 1, 197-203.

[13] Suga, M., Mori, T., Kishimoto, R., Kurokawa, T., Abe, T., Tsuji, H., et al. (2015) Development of a Tissue-Simulating Viscoelastic Gel Phantom for MR Elastography. Proceedings of the European Congress of Radiology, Vienna, 4-8 March.

[14] Yin, M., Rouviere, O., Glaser, K.J. and Ehman, R.L. (2008) Diffraction-Biased Shear Wave Fields Generated with Longitudinal Magnetic Resonance Elastography Drivers. Magnetic Resonance Imaging, 26, 770-780.

[15] Rump, J., Klatt, D., Braun, J., Warmuth, C. and Sack, I. (2007) Fractional Encoding of Harmonic Motions in MR Elastography. Magnetic Resonance Imaging, 57, 388-395. https://doi.org/10.1002/mrm.21152

[16] Klatt, D., Hamhaber, U., Asbach, P., Braun, J. and Sack, I. (2007) Noninvasive Assessment of the Rheological Behavior of Human Organs using Multifrequency MR Elastography: A Study of Brain and Liver Viscoelasticity. Physics in Medicine \& Biology, 52, 7281-7294. https://doi.org/10.1088/0031-9155/52/24/006

[17] Asbach, P., Klatt, D., Hamhaber, U., Braun, J., Somasundaram, R., Hamm, B., et al. (2008) Assessment of Liver Viscoelasticity using Multifrequency MR Elastography. Magnetic Resonance Imaging, 60, 373-379. https://doi.org/10.1002/mrm.21636 